\title{
Clasificación de patrones anormales de autofluorescencia retiniana en el edema macular diabético
}

\author{
Sergio Hernández-Da Mota, ${ }^{1 *}$ Virgilio Lima-Gómez, ${ }^{2}$ Ernesto Rodríguez-Ayala, ${ }^{3}$ \\ Jorge Jans Fromow-Guerra ${ }^{4}$ y Enrique Alfonso Roig Melo-Granados ${ }^{5}$ \\ ${ }^{1}$ Clínica David, Unidad Oftalmógica, Morelia, Michoacán; ' 2 Secretaría de Salud, Hospital Juárez de México, Servicio de Oftalmología, Ciudad de \\ México; ${ }^{3}$ Universidad Anáhuac Norte, Estado de México; ${ }^{4}$ Asociación para Evitar la Ceguera en México, Hospital "Dr. Luis Sánchez Bulnes", Ciudad \\ de México; ${ }^{5}$ Hospital Civil de Guadalajara, Jalisco. México
}

\section{Resumen}

Introducción: Los pacientes con edema macular diabético pueden presentar alteraciones en la autofluorescencia retiniana, que hasta el momento han sido analizadas más con sistemas de láser de barrido o confocales. Objetivo: Describir y clasificar los patrones anormales de autofluorescencia retiniana en pacientes con edema macular diabético mediante el sistema de autofluorescencia retiniana con cámara de flash. Método: Estudio observacional, retrospectivo, transversal y descriptivo. Se evaluaron imágenes digitales de autofluorescencia retiniana de casos no comparativos con edema macular diabético no tratado, obtenidas y almacenadas con el sistema de cámara de flash. Se evaluó la variabilidad interobservador. Resultados: Se incluyeron 37 ojos de 20 pacientes. La opacidad de medios fue la causa más común de calidad inadecuada de imagen. Se observaron cinco diferentes patrones de autofluorescencia retiniana: disminuida (13\%), normal (40 \%), hiperautofluorescente unifocal (17\%), hiperautofluorescente multifocal (22\%) e hiperautofluorescente en placa (8\%). El coeficiente kappa fue de 0.906 ( $p=0.000$ ). Conclusiones: En pacientes con edema macular diabético se presentan diferentes patrones fenotípicos de autofluorescencia retiniana con los sistemas de cámara de flash. Una clasificación fenotípica más precisa pudiera ayudar a establecer factores pronósticos de pérdida visual o al diseño de ensayos clínicos relativos a edema macular diabético.

PALABRAS CLAVE: Autofluorescencia retiniana. Edema macular diabético. Retinopatía diabética.

\begin{abstract}
Introduction: Patients with diabetic macular edema can develop fundus autofluorescence alterations; thus far, these alterations have been more widely studied with scanning or confocal laser systems. Objective: To describe and classify fundus autofluorescence abnormal patterns in patients with diabetic macular edema using the fundus autofluorescence system with a flash camera. Method: Observational, retrospective, cross-sectional, descriptive study. Fundus autofluorescence digital images of non-comparative cases with untreated diabetic macular edema, obtained and stored with a flash camera system, were assessed. Inter-observer variability was evaluated. Results: 37 eyes of 20 patients were included. Lens opacity was the most common cause of inadequate image quality. Five different fundus autofluorescence patterns were observed: decreased (13\%), normal (40\%), focal hyper-autofluorescent (17\%), multi-focal hyper-autofluorescent (22\%) and plaque-like hyper-autofluorescent (8\%). The kappa coefficient was $0.906(p=0.000)$. Conclusions: Different fundus autofluorescence phenotypic patterns are observed with flash camera systems in patients with diabetic macular edema. A more accurate phenotypic classification could help establish prognostic factors for visual loss or for the design of clinical trials for diabetic macular edema.
\end{abstract}

KEY WORDS: Fundus autofluorescence. Diabetic macular edema. Diabetic retinopathy.

Correspondencia:

*Sergio Hernández-Da Mota

E-mail: tolodamota@yahoo.com.mx
Fecha de recepción: 14-01-2019

Fecha de aceptación: 30-05-2019

DOI: 10.24875/GMM.19004992
Gac Med Mex. 2019;155:493-498

Disponible en PubMed

www.gacetamedicademexico.com 


\section{Introducción}

El edema macular diabético (EMD) constituye una de las principales causas de discapacidad visual en el mundo y la principal causa de baja de visión en las personas que padecen diabetes mellitus. ${ }^{1-3}$

La tomografía de coherencia óptica (TCO) y la fluorangiografía se han empleado para la evaluación y el seguimiento terapéutico del EMD., ${ }^{3,4}$ Un estudio de reciente advenimiento y aplicación en la evaluación de los pacientes con EMD es la autofluorescencia retiniana (AFR), un método diagnóstico de la retina que se utiliza como parte de la evaluación integral o multimodal de enfermedades como la degeneración macular relacionada con la edad. ${ }^{5,6}$

Los pacientes con EMD también pueden presentar alteraciones en la AFR, las cuales se han estudiado más con los sistemas de láser de barrido o confocales, no así con los sistemas basados en cámaras de flash. ${ }^{5-15}$ El propósito de este estudio fue describir los patrones de AFR en pacientes con EMD detectado por TCO de dominio espectral.

\section{Método}

Estudio observacional, transversal, descriptivo y retrospectivo, en pacientes con EMD evaluados en la Clínica David, unidad oftalmológica de la Ciudad de Morelia, Michoacán, México, de octubre de 2017 a noviembre de 2018. Se tuvo acceso a 280 pacientes. La investigación se realizó de acuerdo con el código de Núremberg y los principios de la Declaración de Helsinki, y con apego a los lineamientos establecidos en las normas oficiales mexicanas NOM-004 y NOM-012 de la Secretaría de Salud.

Se incluyeron pacientes de uno u otro sexo y de cualquier edad, con diabetes tipo 2, que presentaron EMD detectado clínicamente y por TCO, a quienes se les había realizado estudio de AFR de adecuada calidad. Se excluyeron los pacientes con enfermedades intercurrentes como catarata significativa, degeneración macular relacionada con la edad y quienes habían recibido tratamiento antiangiogénico intravítreo o laserterapia.

Del expediente se obtuvo la información relativa a agudeza visual mejor corregida, sensibilidad al contraste y los hallazgos por fluorangiografía y TCO, que se miden en la clínica conforme a los siguientes procedimientos estandarizados:
- Agudeza visual mejor corregida y sensibilidad al contraste: se midió con la cartilla ETDRS (Early Treatment Diabetic Retinopaty) modificada (Precision Vision, LaSalle, Illinois, Estados Unidos) a una distancia de $4 \mathrm{~m}$, bajo refracción subjetiva. La sensibilidad al contraste se midió empleando la cartilla de sensibilidad al contraste de Hamilton-Veale (Hamilton Veale, Canterbury, Nueva Zelanda).

- Fotografía de fondo, fluorangiografía y AFR: se obtuvieron con una cámara de fondo de flash (cámara de fondo Visucam ${ }^{\mathrm{NM} / \mathrm{FA}}$, Carl Zeiss Meditec, Oberkochen, Alemania). Se tomaron varias fotografías y se descartaron las que tenían artefactos como sombras; las imágenes digitales seleccionadas se guardaron en un disco duro para su posterior análisis, clasificación y procesamiento.

- TCO: a cada paciente se le realizó un escaneo de TCO de dominio espectral (modelo Cirrus 5000 SD-OCT, Carl Zeiss Meditec, Oberkochen, Alemania). De los estudios almacenados, se registró grosor foveal central, volumen del promedio del cubo macular y grosor promedio macular, así como los patrones de EMD: quístico, espongiforme (no quístico) y desprendimiento neurorretiniano seroso.

La variable primaria de resultado fue el patrón de AFR en el área foveal de cada ojo. Dos observadores expertos evaluaron las imágenes de AFR, para lo cual se empleó la siguiente clasificación (Figura 1):

- Patrón de AFR disminuida o hipoautofluorescente.

- Patrón normoautofluorescente o de AFR normal.

- Patrón hiperautofluorescente o de AFR aumentada unifocal.

- Patrón hiperautofluorescente o de AFR aumentada multifocal.

- Patrón hiperautofluorescente o de AFR aumentada en placa.

Se identificaron los porcentajes e intervalos de confianza de cada patrón de autoflurescencia en la muestra, se determinó el coeficiente kappa para evaluar la variabilidad interobservador y se consideró significativo un valor de $p<0.05$.

\section{Resultados}

Se evaluaron 40 ojos de 23 pacientes (edad promedio de $62.8 \pm 7.2$ años); tres ojos de tres pacientes se descartaron por mala calidad de la imagen, debido a de catarata significativa. Se incluyeron 37 ojos de 20 

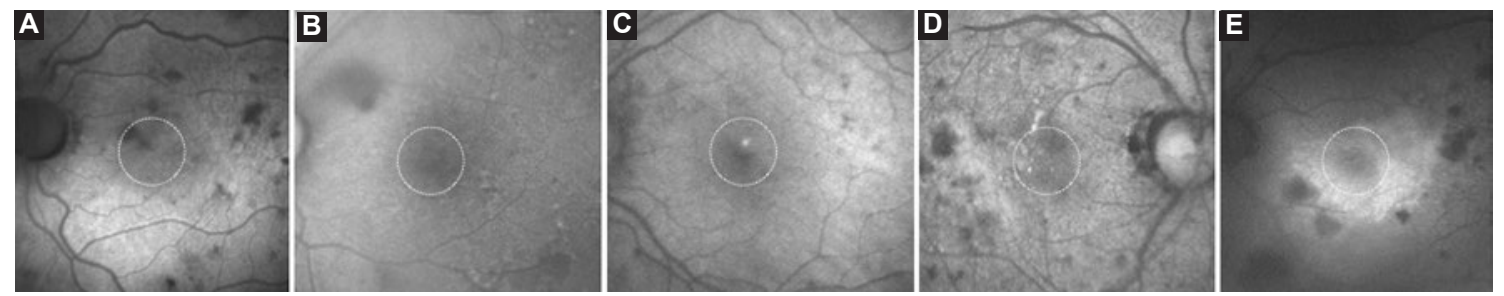

Figura 1. Patrones de autofluorescencia retiniana (AFR) en el área foveal: A: AFR disminuida o hipoautofluorescencia; B: AFR normal o normoautofluorescencia; C: AFR aumentada o hiperautofluorescencia unifocal; D: AFR aumentada o hiperautofluorescencia multifocal; E: AFR aumentada o hiperautofluorescencia en placa.

Tabla 1. Características generales de los pacientes en la muestra y patrón de autofluorescencia retiniana

\begin{tabular}{|c|c|c|}
\hline Característica & $\mathrm{n}$ & $\%$ \\
\hline $\begin{array}{l}\text { Sexo } \\
\text { Masculino } \\
\text { Femenino }\end{array}$ & $\begin{array}{c}12 \\
8\end{array}$ & $\begin{array}{l}60 \\
40\end{array}$ \\
\hline $\begin{array}{l}\text { Patrón de AFR } \\
\text { Hipoautofluorescencia } \\
\text { Normoautofluorescencia } \\
\text { Hiperautofluorescencia unifocal } \\
\text { Hiperautofluorescencia multifocal } \\
\text { Hiperautofluorescencia en placa }\end{array}$ & $\begin{array}{c}5 \\
15 \\
6 \\
8 \\
3\end{array}$ & $\begin{array}{c}13 \\
40 \\
17 \\
22 \\
8\end{array}$ \\
\hline $\begin{array}{l}\text { Patrón de edema por fluorangiografía } \\
\text { Focal } \\
\text { Difuso }\end{array}$ & $\begin{array}{c}8 \\
19\end{array}$ & $\begin{array}{l}30.8 \\
69.2\end{array}$ \\
\hline $\begin{array}{l}\text { Isquemia foveal } \\
\text { Presente } \\
\text { Ausente }\end{array}$ & $\begin{array}{c}9 \\
21\end{array}$ & $\begin{array}{l}32 \\
68\end{array}$ \\
\hline $\begin{array}{l}\text { Patrones de edema por TCO } \\
\text { Quístico } \\
\text { No quístico (espongiforme) } \\
\text { Despegamiento seroso de } \\
\text { neuroretina subfoveal }\end{array}$ & $\begin{array}{c}8 \\
21 \\
6\end{array}$ & $\begin{array}{c}22.9 \\
60 \\
17.1\end{array}$ \\
\hline & Promedio \pm DE & Rango \\
\hline Edad (años) & $62.8 \pm 7.2$ & $53-59$ \\
\hline $\begin{array}{l}\text { AVMC, número de letras vistas por la } \\
\text { cartilla ETDRS }\end{array}$ & $31.5 \pm 16$ & \\
\hline SC, número de pares de letras vistas & $7 \pm 3.5$ & \\
\hline Grosor foveal central por TCO $(\mu \mathrm{m})$ & $356.3 \pm 123.5$ & \\
\hline $\begin{array}{l}\text { Volumen promedio macular por } \\
\text { TCO }\left(\mathrm{mm}^{3}\right)\end{array}$ & $10.9 \pm 3$ & \\
\hline $\begin{array}{l}\text { Grosor promedio macular por } \\
\text { TCO }(\mu \mathrm{m})\end{array}$ & $363.9 \pm 93.9$ & \\
\hline
\end{tabular}

AVMC = agudeza visual mejor corregida, ETDRS = Early Treatment Diabetic Retinopaty $\mathrm{SC}=$ sensibilidad al contraste, $\mathrm{TCO}=$ tomografía de coherencia óptica.

pacientes en total; las características generales de la muestra se describen en la Tabla 1.

Los siguientes patrones de AFR foveal se detectaron en la evaluación fotográfica:

- Hipoautofluorescencia (AFR disminuida): en cinco ojos (13.51\%, IC $95 \%=2.50-24.53)$ se detectó un patrón hipoautofluorescente, sobre todo en la zona periférica de la fóvea y en la zona parafoveal (Figura 1A).

- Normal (AFR normal): en 15 ojos (40.54 \%, IC $95 \%=24.72-53.76)$ de detectó un patrón de AFR de características normales, es decir, sin áreas hiper o hipoautofluorescentes dentro del área foveal (Figura 1B).

- Hiperautofluorescencia unifocal (AFR aumentada unifocal): en seis ojos (16.22 \%, IC $95 \%=4.34$ 28.09). En este tipo de patrón se detectó dentro del área foveal un solo punto de diferente tamaño, con AFR aumentada respecto al resto de dicha área (Figura 1C).

- Hiperautofluorescencia multifocal (AFR aumentada multifocal): en ocho ojos (21.62 \%, IC $95 \%=8.36$ 34.89). En este tipo se encontraron múltiples puntos hiperautofluorescentes dentro del área foveal. Algunos puntos hiperautofluorescentes correspondieron a exudados duros, sobre todo en los de aspecto más refringente de las fotos clínicas ( Figuras 1D y 2).

- Hiperautofluorescencia en placa (AFR aumentada en placa): se observó en tres ojos (8\%, IC $95 \%=0-16.90)$. En este tipo de patrón se apreció un área uniforme hiperautofluorescente dentro del área foveal (Figura 1E).

La distribución de los patrones de AFR en la muestra se presenta en la Figura 3. Los intervalos de confianza señalan que los patrones de hipoautofluorescencia e hiperautofluorescencia en placa fueron consistentemente menos frecuentes que el patrón de AFR normal. El coeficiente kappa para evaluar la concordancia interobservador fue de $0.911(p<0.001)$.

\section{Discusión}

Se ha demostrado que la AFR es una herramienta útil para la detección y monitoreo de diversas entidades retinianas como el EMD, los trastornos retinianos 


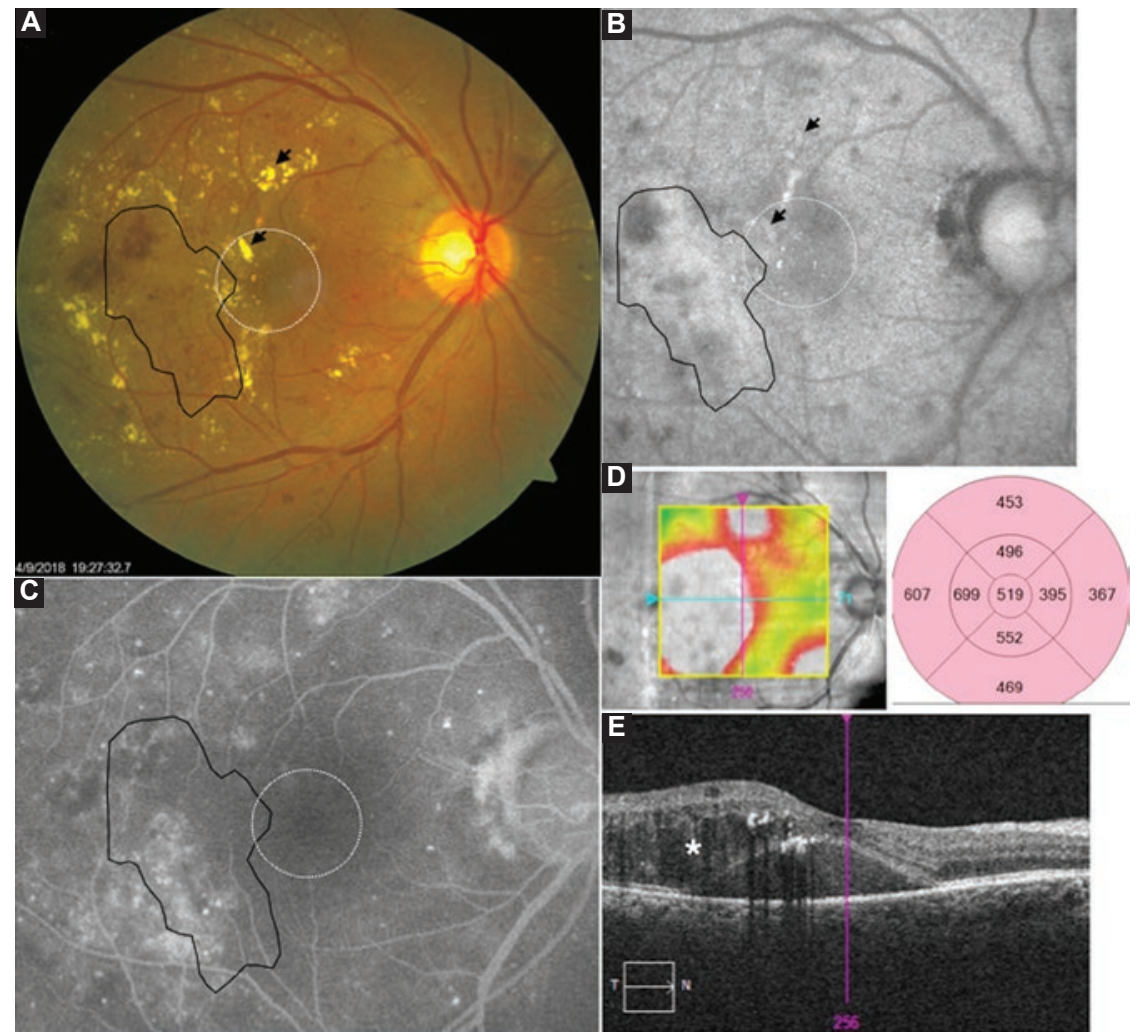

Figura 2. Caso ilustrativo de EMD con patrón foveal de hiperautofluorescencia multifocal (círculo blanco punteado) y autofluorescencia retiniana (AFR) en placa extrafoveal (zona delineada de negro). A: Fotografía clínica de polo posterior; las fechas indican exudados duros; B: Las flechas indican AFR aumentada, que se corresponde con los exudados de la foto clínica. La zona delineada de negro indica un área de AFR aumentada con aspecto de placa, extrafoveal, que se corresponde con la zona de fuga en la fluorangiografía en la imagen C; C: Se corresponde también con la zona de engrosamiento de la mácula en la imagen infrarroja con montaje de grosor en color de la tomografía de coherencia óptica en la imagen $\mathbf{D}$ y se corresponde con la zona que tiene presencia de quistes y despegamiento seroso de neurorretina subfoveal (*) en la imagen $\mathbf{E}$.

hereditarios y la degeneración macular relacionada con la edad. ${ }^{7}$

En el EMD, si bien la AFR ha demostrado su utilidad, su uso ha estado más limitado y se ha difundido poco en la comunidad de médicos dedicados al tratamiento de la patología macular. ${ }^{7}$

Varios autores ${ }^{8-15}$ han descrito diferentes patrones de cambio en la AFR en los pacientes que padecen de EMD. Pece et al. ${ }^{15}$ describieron dos tipos:

- Tipo 1, al que denominaron multilobulado, caracterizado por múltiples áreas de AFR aumentada, similar al de hiperautofluorescencia multifocal de nuestra clasificación.

- Tipo 2 o unilobulado, que corresponde a un gran quiste en la imagen de TCO.

- Tipo 3 o mixto, con características combinadas de los tipos 1 y 2.

Vujosevic et al.12 describieron una clasificación con los siguientes patrones de AFR dentro del área foveal: normal, autofluorescencia aumentada unifocal y autofluorescencia aumentada multifocal. En ese estudio, los autores emplearon un sistema de láser de barrido confocal para obtener las imágenes de AFR y consideraron que los casos que tuvieron AFR disminuida fueron el resultado del bloqueo que producen los pigmentos maculares sobre la AFR, por lo que los consideraron normales.

En la AFR obtenida mediante los sistemas de cámara de flash, tanto la excitación como la emisión de la señal de AFR ocurren en una longitud de onda mayor, lo que se traduce en un aspecto de menor densidad de los pigmentos maculares en la imagen. Hubo casos en los que la AFR disminuyó más de lo que consideramos un patrón normal, sobre todo en la periferia de la fóvea y en la parafóvea, lo cual aparentemente no fue causado por los pigmentos. Por esta razón modificamos la clasificación descrita por Vujosevic et al. ${ }^{12}$ y agregamos un nuevo patrón: AFR disminuida. Este patrón podría deberse a la presencia de un aumento del grosor retiniano en la periferia del área foveal y debe considerarse una categoría separada de AFR foveal.

Encontramos tres casos con gran placa o área de AFR aumentada, que clasificamos en el patrón hiperautofluorescente o AFR aumentada en placa. Creemos que el aspecto de este patrón se debe a la 


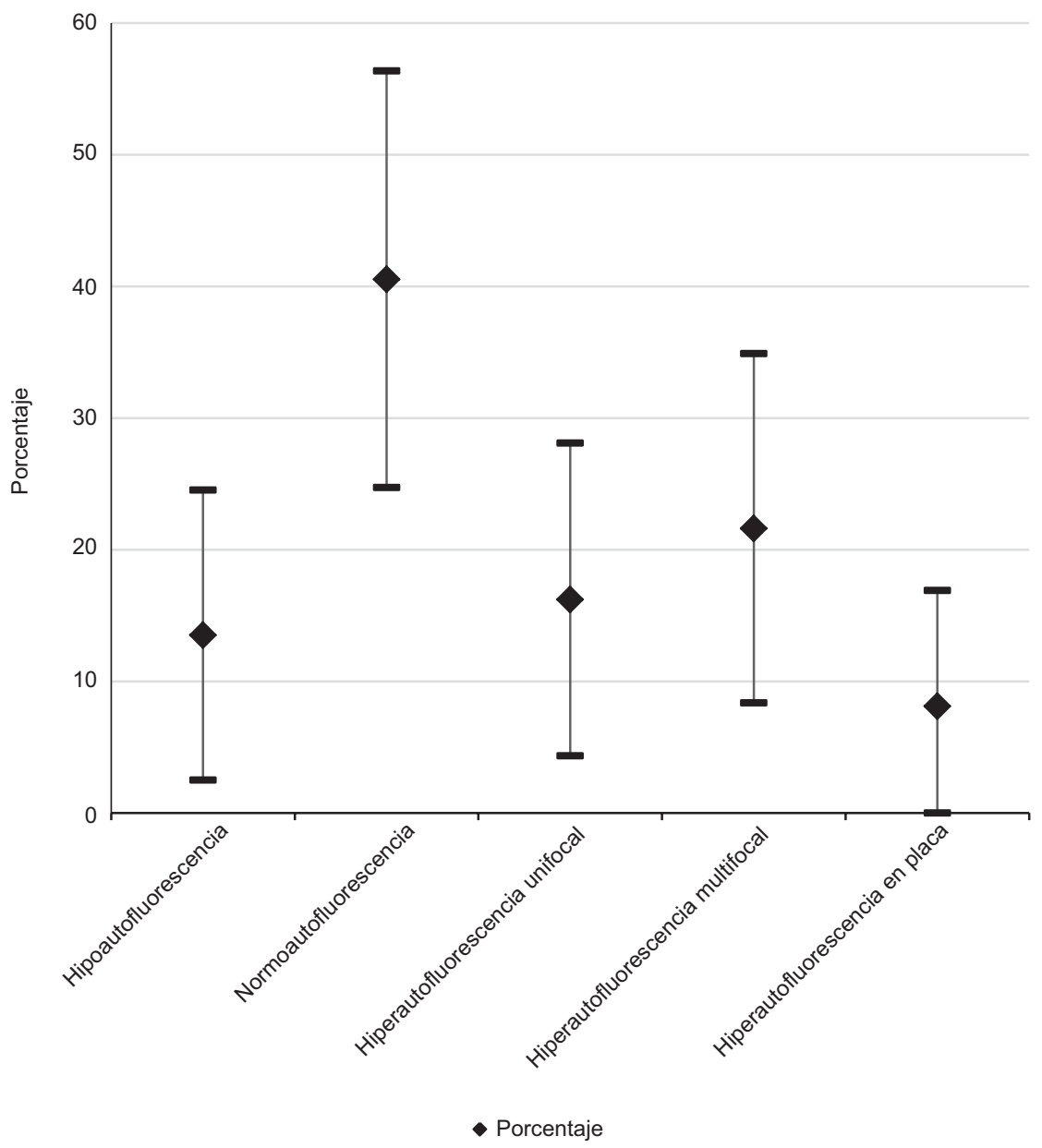

Figura 3. Distribución porcentual e intervalos de confianza de $95 \%$ de los patrones de autofluorescencia en la muestra.

confluencia de numerosos quistes intrarretinianos, lo que aunado al bajo contraste en las imágenes de AFR que tienen los sistemas de cámara de flash da la apariencia de una gran placa o zona difusa de hiperautofluorescencia en el área foveal.

Otro hallazgo interesante fue que algunos puntos con AFR aumentada, sobre todo en los casos con un patrón de AFR aumentada uni y multifocal, en la foto clínica correspondían con algunos exudados de aspecto más refringente.

Chung et al. ${ }^{10}$ describieron un sistema de evaluación de la AFR foveolar:

- Grado 1, ojos sin AFR aumentada en la foveola.

- Grado 2, ojos con AFR aumentada de menos de la mitad del área foveolar.

- Grado 3, ojos con AFR aumentada entre la mitad y tres cuartos del área foveolar.

- Grado 4, cuando la AFR aumentada ocupaba la totalidad del área foveolar.

La clasificación de los diferentes patrones de AFR, sobre todo con los sistemas confocales, ha servido para diseñar estudios cuyo propósito principal ha sido evaluar la correlación entre AFR y variables anatomofuncionales retinianas. ${ }^{9-15}$

Respecto a las limitaciones de nuestro estudio, en primer lugar está el uso de una cámara flash para obtener imágenes de AFR. Las cámaras de fondo capturan más luz reflejada y dispersa en comparación con los sistemas confocales; la luz dispersada desde estructuras fuera del plano retiniano puede aumentar artificialmente la señal de AFR, un fenómeno denominado pseudoautofluorescencia. ${ }^{7}$

Las cámaras de fondo también producen imágenes con bajo contraste, lo que puede llevar a inadecuada interpretación de las imágenes debido a la posible confusión de los patrones de AFR foveal. En este sentido, sería interesante evaluar el papel de los métodos cuantitativos de AFR.

En pacientes con EMD se presentan diferentes patrones de AFR con los sistemas de cámara de flash. Una clasificación fenotípica más precisa puede ayudar a determinar factores pronósticos de pérdida 


\section{visual o para el diseño de otros ensayos clínicos para el EMD.}

Por último, la AFR se perfila como un estudio con gran potencial para la evaluación integral de la función retiniana en la era actual, en la que prevalece el paradigma del diagnóstico con base en la imagenología retiniana multimodal.

\section{Bibliografía}

1. Shaw JE, Sicree RA, Zimmet PZ. Global estimates of the prevalence of diabetes for 2010 and 2030. Diabetes Res Clin Pract. 2010;87:4-14.

2. Klein R, Knudtson MD, Lee KE, Gangnon R, Klein BE. The Wisconsin Epidemiologic Study of Diabetic Retinopathy XXIII: twenty-five-year incidence of macular edema in persons with type 1 diabetes. Ophthalmology. 2009;116:497-503

3. Girach A, Lund-Andersen H. Diabetic macular oedema: a clinical overview. Int J Clin Pract. 2007;61:88-97.

4. Acón D, Wu L. Multimodal imaging in diabetic macular edema. Asia Pac J Ophthalmol (Phila). 2018;7:22-27.

5. Midena E, Bini S. Multimodal retinal imaging of diabetic macular edema: toward new paradigms of pathophysiology. Graefes Arch Clin Exp Ophthalmol. 2016;254:1661-1668.
6. Pichi F, Abboud EB, Ghazi NG, Khan AO. Fundus autofluorescence imaging in hereditary retinal diseases. Acta Ophthalmol. 2018;96:e549-e561.

7. Yung M, Klufas MA, Sarraf D. Clinical applications of fundus autofluorescence in retinal disease. Int J Retina Vitreous. 2016:2:12.

8. McBain VA, Forrester JV, Lois N. Fundus autofluorescence in the diagnosis of cystoid macular oedema. Br J Ophthalmol. 2008;92:946-949.

9. Vujosevic S, Torresin T, Bini S, Convento E, Pilotto E, Parrozzani R, et al. Imaging retinal inflammatory biomarkers after intravitreal steroid and anti-VEGF treatment in diabetic macular oedema. Acta Ophthalmol. 2017:95:464-471.

10. Chung H, Park B, Shin HJ, Kim HC. Correlation of fundus autofluorescence with spectral-domain optical coherence tomography and vision in diabetic macular edema. Ophthalmology. 2012;119:1056-1065.

11. Vujosevic S, Bottega E, Casciano M, Pilotto E, Convento E, Midena E. Microperimetry and fundus autofluorescence in diabetic macular edema: subthreshold micropulse diode laser versus modified early treatment diabetic retinopathy study laser photocoagulation. Retina. 2010; 30:908-916.

12. Vujosevic S, Casciano M, Pilotto E, Boccassini B, Varano M, Midena E. Diabetic macular edema: fundus autofluorescence and functional correlations. Invest Ophthalmol Vis Sci. 2011;52:442-448.

13. Shen $Y, X u X$, Liu K. Fundus autofluorescence characteristics in patients with diabetic macular edema. Chin Med J (Engl). 2014;127:1423-1428.

14. Reznicek L, Dabov S, Haritoglou C, et al. Green-light fundus autofluorescence in diabetic macular edema. Int J Ophthalmol. 2013;6:75-80.

15. Pece A, Isola V, Holz F, Milani P, Brancato R. Autofluorescence imaging of cystoid macular edema in diabetic retinopathy. Ophthalmologica 2010;224:230-235 